\title{
Space Charge of Polyethylene and Electronic Structure Analysis of Trapping Site Using Common Chemical Groups
}

\author{
Weiwang Wang, ${ }^{1,2}$ Yasuhiro Tanaka, ${ }^{2 *}$ and Tatsuo Takada ${ }^{2}$ \\ ${ }^{1}$ State Key Laboratory of Electrical Insulation and Power Equipment, School of Electrical Engineering, \\ Xi'an Jiaotong University, Xi'an 710049, China \\ ${ }^{2}$ Measurement and Electric Machine Control Laboratory, Tokyo City University, \\ Tamazutsumi, Setagaya 158-8557, Tokyo, Japan
}

(Received December 7, 2016; accepted June 27, 2017)

Keywords: space charge, PEA, quantum chemical calculation, band structure, trapping site

Pulsed electroacoustic (PEA) measurement technology is widely accepted to detect the space charge distribution inside insulating materials all over the world. In this work, we elucidated further the space charge mechanism based on the typical PEA results of low-density polyethylene (LDPE). Clearly, significant positive accumulation and charge packets are formed inside the sample, which is derived from the chemical and physical structures of polyethylene. Usually, some common chemical groups, such as hydroxyl, carbonyl, double bond, conjugated double bond, and oxide center, exist in polyethylene owing to the manufacturing process and complex ambient. By employing an advanced quantum chemical calculation technique, we obtained the electronic structures of a small polyethylene (PE) chain $\left(\mathrm{C}_{24} \mathrm{H}_{50}\right)$ and some PE chains with different chemical groups. After analyzing the electron energy level, molecular orbital, and electric potential, we determined the trapping sites by the chemical groups. These chemical groups with special structures contribute to the narrowing of the highest occupied molecular orbital (HOMO) and lowest unoccupied molecular orbital (LUMO) gaps (namely, the band gap here), indicating the introduction of trapping levels within the band gap of PE. Additionally, 3D electric potential distributions demonstrate that an obvious potential distortion occurs around these introduced chemical groups. This means that the moving electron or hole carriers can be captured here, leading to the formation of trapped charges. These trapping sites inside the polyethylene are closely related to the space charge accumulation in LDPE.

\section{Introduction}

Understanding space charge behaviors of dielectrics plays a significant role in the design and development of new insulating materials used in power systems. A nondestructive detecting technique is of considerable value in determining space charge properties in dielectrics. Owing to the development of a high-quality pulsed electroacoustic (PEA) system, ${ }^{(1)}$ nothing is more important than using this technique to obtain directly the space charge profiles inside the materials. Using the PEA system, considerable works of space charge characteristics in polymeric materials have been reported. These works make great contributions to the dielectric properties, mechanisms

"Corresponding author: e-mail: ytanaka@tcu.ac.jp

http://dx.doi.org/10.18494/SAM.2017.1553 
and newly designed materials in electrical and other fields. ${ }^{(2,3)}$ In addition, many favorable and useful PEA systems with performance improvements were developed from 20 years ago, such as high-temperature, high-voltage, low-temperature, high-resolution, and mini-PEA systems. ${ }^{(4)}$ Almost 30 years has passed since the development of the first PEA system. The scientific works using PEA systems not only contribute to the space charge behaviors in insulating materials but also benefit the investigation and design of power electronic devices. Furthermore, new methods for clarifying the physical mechanisms underlying the space charge behaviors have been proposed.

Considerable works have pointed out that the space charge under a high electric field is closely related to the charge trapping processes in insulating materials. ${ }^{(2,5)}$ Recently, some researchers have studied the charge trapping in polymeric materials by molecular simulation and calculation. Meunier et al. ${ }^{(6)}$ used molecular simulation to study the electron trapping in polyethylene containing chemical defects. They found the introduction of shallow or deep trap sites by some chemical groups and calculated the corresponding trapping levels. Previous works have studied the charge trapping site in saturated and aromatic polymers by quantum chemical calculation based on the density functional theory (DFT). ${ }^{(7,8)}$ They pointed out that the chemical structures or groups can create trapping sites. From these calculations, the space charge behaviors in lowdensity polyethylene (LDPE), polyimide (Kapton) and ethylene tetrafluoroethylene (ETFE) irradiated by electron beams have been explained. Huzayyin et al. ${ }^{(9,10)}$ have studied the effect of chemical impurities such as carbonyl, vinyl, double groups on dielectric polyethylene. Using density functional analysis, these chemical impurities introduced deep and shallow states between the valence maximum and the conduction band minimum. The energy level formed by carbonyl was $1.63 \mathrm{eV}^{(10)}$ The chemical impurities also enhanced the interchain interaction, which was beneficial to the interchain charge transfer and conduction. Unge et al. ${ }^{(11)}$ have studied the deep traps in polyethylene (amorphous) originating from carbonyl, conjugated double bond (Conju. DB), hydroxyl, triple bond, and vinyl impurities using ab initio simulation. The cross-linking byproducts, such as acetophenone and alpha-methylstyrene, seemed to introduce deeper electron traps $(2.28$ and $1.79 \mathrm{eV}) .^{(11)}$

In this work, we focus on the origin of trapping sites in polyethylene chains by some common chemical impurities. Using the quantum chemical calculation with the DFT method, we obtained the energy level, band structure, molecular orbitals (MOs), and electric potential distributions of a small polyethylene (PE) chain $\left(\mathrm{C}_{24} \mathrm{H}_{50}\right)$ and different molecular structures with chemical groups. After that, we describe the formation of charge trapping after analyzing the electronic structures of different molecular structures. The introduction of trapping sites is of prime importance to understanding the space charge of LDPE. We discuss the influence of the trapping sites by chemical impurities qualitatively for understanding the charge accumulation, especially for the positive charge.

\section{Space Charge Measurement}

\subsection{Typical PEA system}

Figure 1 shows a typical PEA measurement system. It mainly includes three parts: a highvoltage electrode (for applying dc and pulse voltages), a low-voltage electrode (sensor) system, and a signal measurement unit. Silicone oil was used to surround the sample to prevent surface 


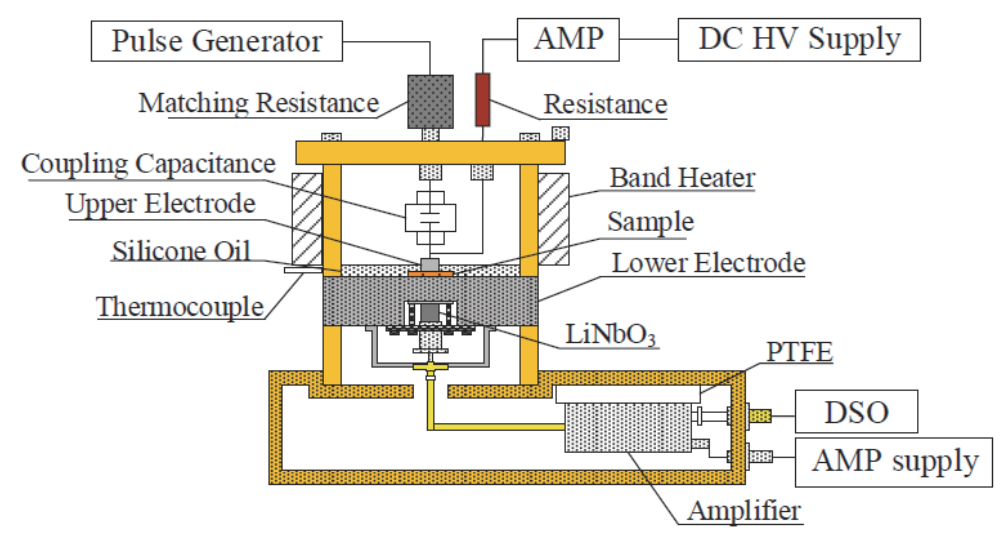

Fig. 1. (Color online) Typical PEA measurement structure.

flashover. Usually, we use a semiconductor (SC) layer (carbon black-filled polymer) between the high-voltage electrode and the measured sample to match the acoustic impedance. When the pulse voltage was applied to the sample, the narrow charge layer or interface charge (induced at the electrode/sample surfaces) would generate pressure waves. These pressure waves propagate through the electrode and sample. When they arrive at the sensor surface, the piezoelectric sensor transforms the signals of the pressure wave originating from the space charge to an output voltage. Finally, the output signals are transmitted to an oscilloscope to record the measured data. After that, we use signal processing, including time-frequency transformations (FFT and IFFT) to obtain the space charge distribution along the sample thickness.

Here, a sensor with a $\mathrm{LiNbO}_{3}$ piezoelectric crystal material was used in the improved PEA system. This sensor material can be used under a high temperature. A pulse generator with an amplitude of $500 \mathrm{~V}$ and a pulse width of $5 \mathrm{~ns}$ was also used. The resolution of this PEA system is about $12 \mu \mathrm{m}$ for a polymeric material according to the response of the sensor and the pulse width. This PEA system has advantages for measuring thin films under different temperatures.

\subsection{Space charge of LDPE}

A LDPE film of $100 \mu \mathrm{m}$ thickness was measured here. The time dependence of the space charge distribution was determined under a high electric stress. An electric field of $100 \mathrm{kV} / \mathrm{mm}$ was applied to the sample and the space charge profiles were recorded for $3 \mathrm{~h}$.

Figure 2 shows the space charge dynamics of the LDPE film along the sample thickness. The positive charge (hole) injection from the SC electrode is observed under $100 \mathrm{kV} / \mathrm{mm}$. Additionally, a positive charge packet is formed and moves toward the cathode (Al electrode) over time. It moves rapidly within $1 \mathrm{~h}$ after applying voltage, while the speed of the charge packet decreases after 1 $\mathrm{h}$ and gradually becomes stable at a certain position near the cathode, as shown in Fig. 2. The packet charge has a high charge density of $500 \mathrm{C} / \mathrm{m}^{3}$, indicating large amounts of positive charge accumulation inside the LDPE sample. However, electron injection from the cathode is difficult, and slight negative charge accumulation occurs inside the LDPE film. Even if the negative charge injection occurs when the electric field is very high near the cathode (Fig. 2), the amounts of negative charges inside the sample significantly decrease owing to the recombination with the positive charges. This leads to less negative charge accumulation inside the sample. 


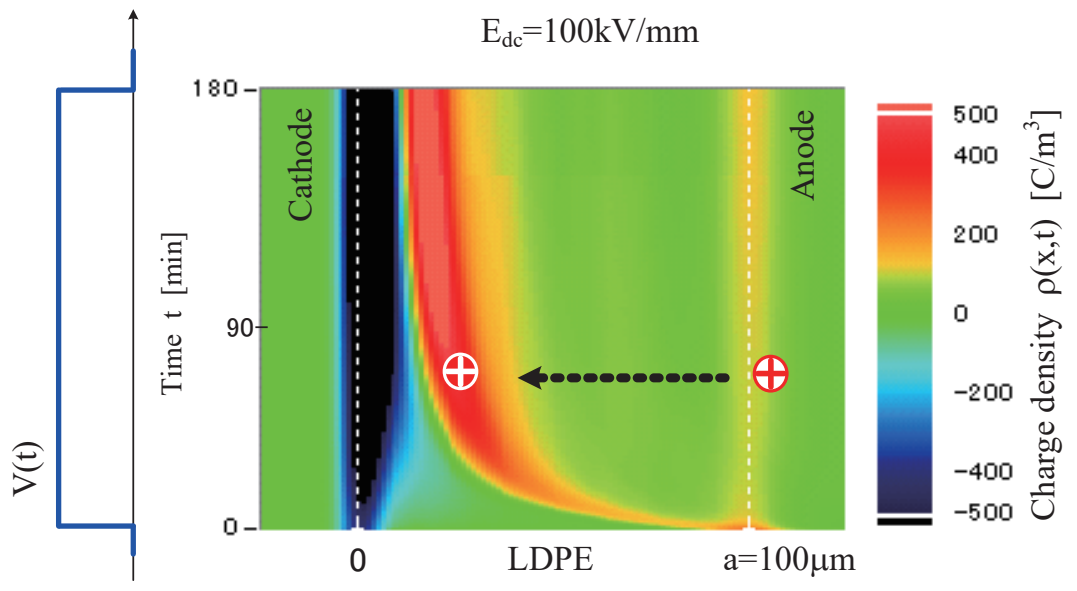

Fig. 2. (Color online) Space charge of LDPE under high electric field.

The significant positive charge packet has been observed in LDPE and cross-linked polyethylene (XLPE) films under high electric fields. ${ }^{(12,13)}$ It is accepted that the charge trapping and detrapping processes dominate the space charge in polyethylene materials. This will give rise to the formation of different carrier mobility or conductivity regions, leading to the generation of charge packets.

\section{Quantum Chemical Calculation}

\subsection{Calculation methodology}

From the above space charge result and analysis, it is necessary to study the trapping and detrapping mechanisms in polyethylene dielectrics. In general, the polymeric material has many types of traps due to the complex physical and chemical structures. ${ }^{(2,14)}$ Here, we conduct the advanced quantum chemical technique to study the influence of chemical impurities on trapping sites in a polyethylene chain.

A small molecular PE chain $\left(\mathrm{C}_{24} \mathrm{H}_{50}\right)$ is built on the basis of the ground-state configuration of electrons and nuclei of carbon $(\mathrm{C})$ and hydrogen $(\mathrm{H})$. The state can be determined by combining the DFT and the optimization of the molecular structure according to the energy minimization. In this work, the energy levels and MOs were calculated using Gaussian 09 and Gaussian view software. The DFT with the B3LYP hybrid functional and 6-31G basis function was selected to calculate the wave functions of molecules. The calculation method was also described in a previous work. ${ }^{(8)}$ These basis and calculation functions determine the electronic eigenvalues (energy levels of molecular orbitals) and wave functions. According to these results, various characteristics such as energy level, band structure, electron density, dipole moment, and electric charges are obtained. This work employs some chemical impurities to replace the hydrogen atoms or enter the main $\mathrm{C}-\mathrm{C}$ backbone. In this case, we built some dielectric molecular structures. After that, we use the calculation methods described above to obtain the electronic structures and various properties. 


\subsection{Band structure and energy level}

Figure 3 shows the calculated electronic structures of different molecular chains. It is clearly observed that the introduction of these chemical groups changes the energy level distributions. The band gap $\varphi_{g}$ is the difference between the highest occupied molecular orbital (HOMO) and lowest unoccupied molecular orbital (LUMO) levels. The band gap of $\mathrm{PE}\left(\varphi_{g}\right)$ is $9.99 \mathrm{eV}$, which is higher than that of the published value of $8.8 \mathrm{eV} .^{(15,16)}$ This is probably caused by the small size of this PE chain. In addition, the LUMO level is lower than the vacuum level (VL), which means that a negative affinity exists in PE. The published work also verified this negative affinity in PE. ${ }^{(17)}$

After introducing the chemical groups, the LUMO level starts to decrease. The LUMO level of the hydroxyl group chain is $+1.77 \mathrm{eV}$, which is slightly lower than that of the PE chain. This LUMO level locates above the VL like the PE chain. There exists a small gap between the LUMO level and its higher level, as shown in Fig. 3. After employing the carbonyl in the PE chain, the LUMO level decreases further and locates very close to the VL. In this case, the gap between the LUMO level and its higher level becomes larger than that of the hydroxyl group chain. When introducing the oxygen and Conju. DB, the LUMO level continues to decrease and becomes lower than the VL [e.g., $-0.51 \mathrm{eV}$ for the oxygen center group $\left(\mathrm{C}_{24} \mathrm{H}_{48} \mathrm{O}\right)$ and $-0.27 \mathrm{eV}$ for the Conju. $\mathrm{DB}\left(\mathrm{C}_{24} \mathrm{H}_{46}\right)$ ]. The HOMO level presents an obvious increase in comparison with that of the PE chain, as shown in Fig. 3. For example, the HOMO level of the Conju. DB is $-5.62 \mathrm{eV}$, which is higher than that of the PE chain $(-7.6 \mathrm{eV})$. As a result, the gap $\left(\varphi_{g}\right)$ significantly decreases $[5.35 \mathrm{eV}$ for the Conju. DB]. This also indicates that a large energy gap occurs between the HOMO level and the lower level, especially for the Conju. DB chain.

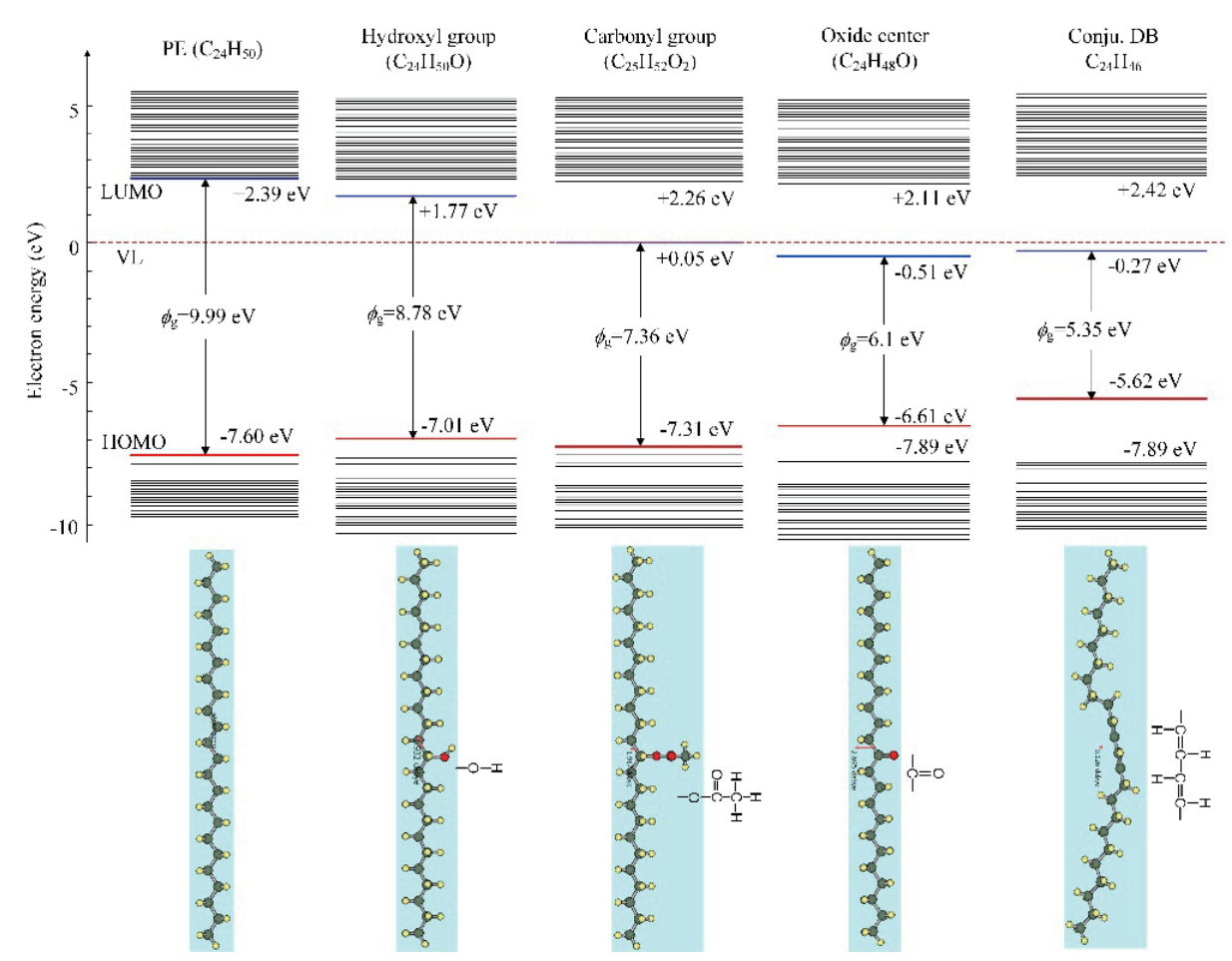

Fig. 3. (Color online) Band structures and calculated energy levels; the red and blue lines are HOMO and LUMO levels, respectively. $\varphi_{g}$ is the HOMO-LUMO gap; VL is the vacuum level. 
Figure 4 shows the curves of energy levels as a function of molecular structures. It can be seen clearly that $\varphi_{g}$ generally decreases after introducing the chemical groups. $\varphi_{g}$ can be reduced by $46 \%$ in the Conju. DB molecular structure compared with that of the PE chain. This reduction is ascribed to the significant decrease in LUMO level and the increase in HOMO level by Conju. DB. It can be clearly concluded that some new energy levels are created within the band gap of the PE chain by these chemical groups, and also some energy gaps exist near the HOMO and LUMO energy levels. These variations of electron energy levels play important roles in the band structure of PE.

Figure 5 shows the calculated energy levels and related MOs of some molecular structures. For the PE chain, the orbital electrons present a high probability of distribution along the chain at the HOMO level $(-7.60 \mathrm{eV})$, as shown in Fig. 5(a). However, MOs are strongly localized within the chain (between $\mathrm{C}-\mathrm{H}$ bonds) at the LUMO level $(+2.39 \mathrm{eV})$. This indicates that orbital electrons have low probabilities to distribute the LUMO level. From the previous work, ${ }^{(8)}$ it can be concluded that this HOMO level can be regarded as the conduction level for hole carriers, and this LUMO level is the conduction level for electrons.

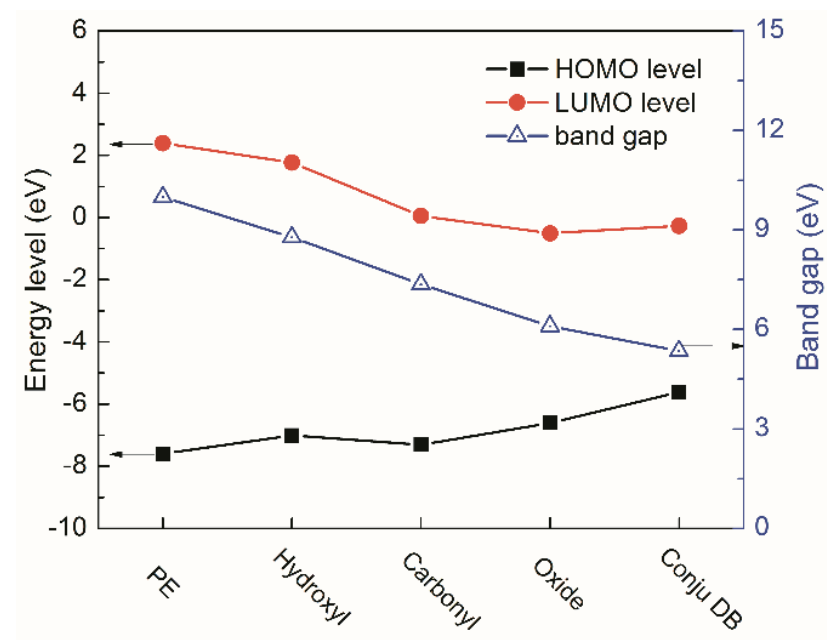

Fig. 4. (Color online) Variations of energy levels and band gaps for dielectric molecular structures.

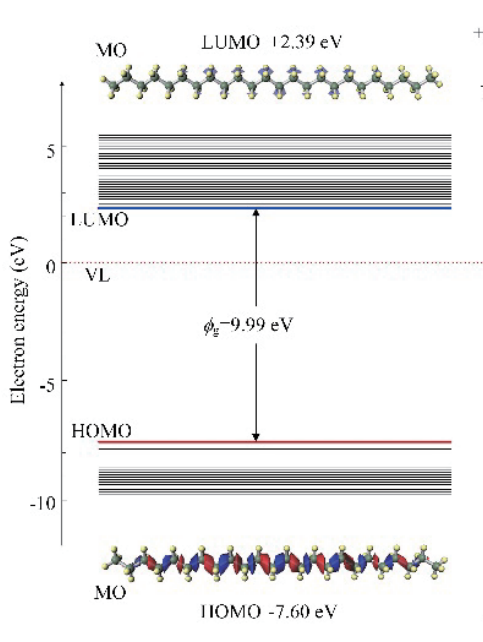

(a)

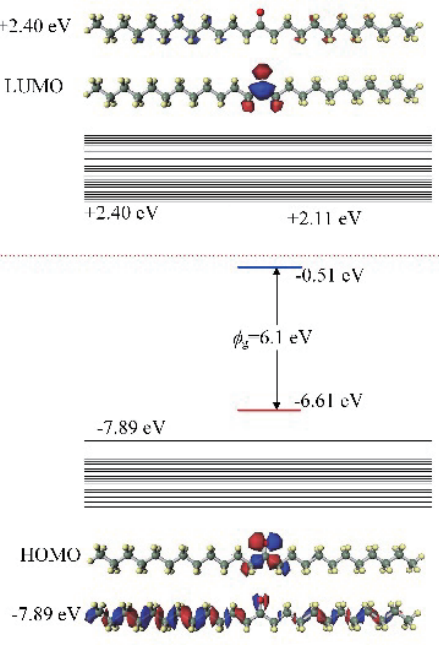

(b)

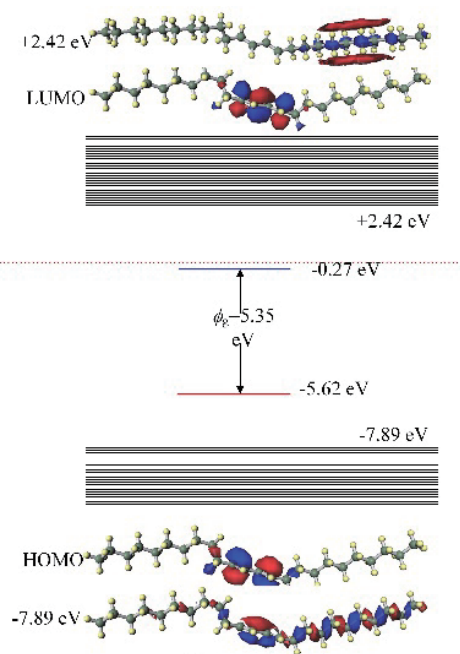

(c)

Fig. 5. (Color online) Molecular orbitals of (a) PE, (b) oxide center, and (c) Conju. DB molecular structures. 
As for the oxide center chain $\left(\mathrm{C}_{24} \mathrm{H}_{48} \mathrm{O}\right)$, the orbital electrons present a high probability of local distribution around the $\mathrm{C}=\mathrm{O}$ group at the HOMO and LUMO levels ( $\mathrm{MO}$ is localized at the $\mathrm{C}=\mathrm{O}$ group), as shown in Fig. 5(b). In this situation, the LUMO level $(-0.51 \mathrm{eV})$ is slightly lower than the VL. A large gap exists between the LUMO level and the higher level $(+2.11 \mathrm{eV})$. However, the energy levels above the LUMO level present a continuous distribution with small energy difference. For the $+2.40 \mathrm{eV}$ level, the MO is similar to the LUMO level of the PE chain, which indicates the bottom of the conduction band. Similarly, a large gap also occurs between the HOMO level and the level below $-7.89 \mathrm{eV}$. The orbital electrons are likely to distribute along the chain at $-7.89 \mathrm{eV}$ below the HOMO level, which is similar to the HOMO level of the PE chain. The energy levels below the HOMO levels can be regarded as the valence band.

\subsection{Electric potential distribution}

To further study the effects of these new energy levels, we calculated the electric potential distributions of the molecular structures according to the Mulliken charges of each atom and its coordinates $(x, y, z)$. After that, the electric potential of the molecular structure is given by

$$
V(x, y, z)=\sum_{i=1}^{n} \frac{q_{i}\left(x_{i}, y_{i}, z_{i}\right)}{4 \pi \varepsilon_{0} \sqrt{\left(x_{i}-x\right)^{2}+\left(y_{i}-y\right)^{2}+\left(z_{i}-z\right)^{2}}},
$$

where $q_{i}\left(x_{i}, y_{i}, z_{i}\right)$ is the electric charge (Mulliken atomic charges) of each atom and its coordinates (standard orientation), and $\varepsilon_{0}$ is the permittivity of free space. All the results were calculated using small molecular chains in a vacuum. Hence, we neglect the permittivity of the material $\left(\varepsilon_{r}\right)$.

Figure 6 shows the 3D electric potential distribution $V(x, y, z)$ maps of the molecular structures. Both positive and negative potentials are distributed along the molecular chains. For the PE chain, the carbon atom is charged negatively and the hydrogen atom is charged positively because

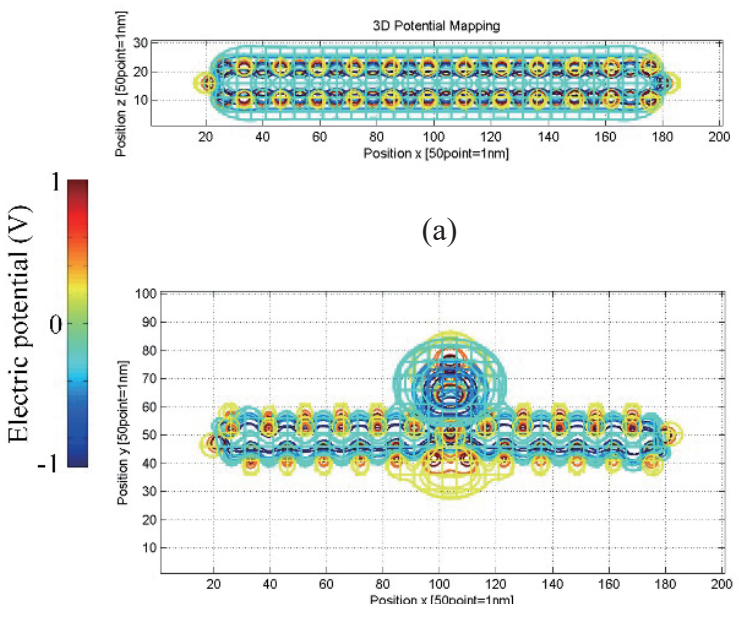

(c)

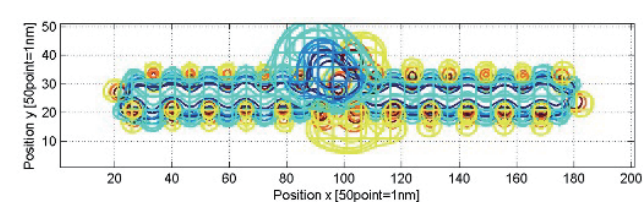

(b)

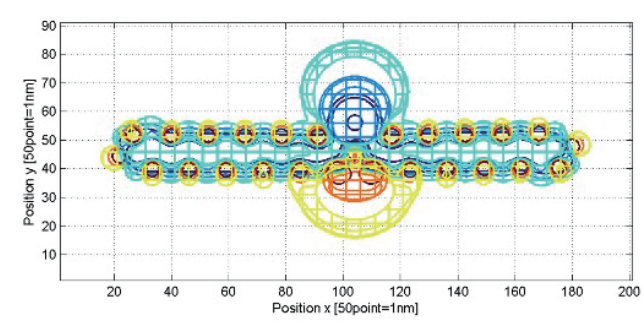

(d)

Fig. 6. (Color online) Electric potential distribution maps of different molecular structures. (a) PE, $\mu=0$ debye; (b) hydroxy group, $\mu=1.932$ debye; (c) carbonyl group, $\mu=1.917$ debye; and (d) oxygen center, $\mu=2.695$ debye. 
the electronegativity of carbon (2.5) is higher than that of hydrogen (2.1). There is no potential distortion along the small PE chain. However, after introducing the chemical groups, the electric potentials become clearly distorted at the position of the chemical groups, as shown in Fig. 6. For the hydroxyl group chain, the large negative potential appears around the oxygen atom because of its high electronegativity (3.5). The positive potential around the hydrogen atoms also increases. If the electron or hole carriers move to the place near the hydroxyl group, they are likely to be attracted or captured here. In the case of the oxide center chain, it can be observed that a high negative potential exists around oxygen, and also a large positive potential occurs opposite the $\mathrm{C}=\mathrm{O}$ group (around hydrogen atoms). The carbon atom that connected to the oxygen becomes positively charged because of the high electronegativity of oxygen. The electric potentials around this $\mathrm{C}=\mathrm{O}$ group present much higher values than these of the PE chain and hydroxyl group structure.

\section{Discussion of Trapping Site}

It is evident to infer from the above descriptions that the chemical groups introduced into the $\mathrm{PE}$ chain $\mathrm{C}_{24} \mathrm{H}_{50}$ create new localized states, which refers to some new energy levels that located within the band gap of the PE chain. Firstly, these new energy levels reduce the band gap of the PE chain. This is beneficial for charge excitation from the HOMO to LUMO levels (new energy levels) or from these levels to above LUMO or below LUMO levels (conduction and valence bands). This is also necessary for electron and hole hopping from these localized states to conduction and valence bands with the required energy. Hence, it is reasonable that these new energy levels can be regarded as the trapping sites for hindering charge transport at conduction and valence band edges and forming the hopping conduction between the localized states (shallow traps).

Secondly, the MOs near the HOMO and LUMO levels are localized at the positions of these chemical impurities. It turns out that the electrons and holes that locate at these levels are difficult to detrap, indicating the presence of some trapping sites here. Finally, based on the electric potential distributions, it can be confirmed that these chemical groups generate an electric potential distortion that served as the trapping site to capture electron and hole carriers. The trapping site is closely related to the electric dipole of these chemical structures, as shown in the dipole moment $(\mu)$ in Fig. 6.

The created trapping sites are located at these chemical group positions and acted as deep or shallow trap centers. It can be concluded that the created new energy levels near the HOMO and LUMO correspond to the hole and electron traps, respectively. With respect to the hydroxyl group, deep traps can be easily introduced by the carbonyl, oxygen center, and Conju. DB. The estimated deep traps $(2-2.5 \mathrm{eV})$ introduced by the carbonyl and Conju. DB groups can fit the results published by Huzayyin et al. ${ }^{(9,10)}$ and Unge et al. ${ }^{(11)}$

These trapping sites play important roles in the space charge of LDPE. For positive charge accumulation, the hole traps introduced by the carbonyl, oxide, and Conju. DB groups act as deep traps to capture the hole carriers, resulting in positive charge accumulation. For negative charge accumulation, a previous work pointed out that the charge injection is difficult to occur owing to the high electron barrier, ${ }^{(7)}$ and even the electron traps can be generated by these chemical groups. 


\section{Conclusions}

The introduction of common chemical groups into the $\mathrm{PE}$ chain $\mathrm{C}_{24} \mathrm{H}_{50}$ decreased the HOMOLUMO gap and created new energy levels. Considering the electronic structure, the energy level, MOs, and electric potential distributions, it can be concluded that these chemical groups created new charge trapping sites in the PE chain owing to the nature of its electronegativity of the atom and the electric dipole moment. These created new trapping sites located at the positions of the introduced chemical groups, which acted as either deep or shallow traps within the PE band gap.

These introduced trapping sites behave as some trap centers in PE, which can capture the electron or hole carriers. This leads to space charge accumulation inside the LDPE sample. Positive charge accumulation was ascribed to the deep hole trap sites. However, slight negative charge accumulation was derived from the restricted electron injection or charge recombination.

\section{References}

1 T. Takada: IEEE Trans. Dielectr. Electr. Insul. 6 (1999) 519.

2 G. Teyssedre and C. Laurent: IEEE Trans. Dielectr. Electr. Insul. 12 (2005) 857.

3 H. Naohiro, T. Gilbert, L. Christian, and F. Kaori: J. Phys. D: Appl. Phys. 37 (2004) 1327.

4 K. Fukunaga: IEEE Electr. Insul. Mag. 20 (2004) 18.

5 G. Chen and J. Zhao: J. Phys. D: Appl. Phys. 44 (2011) 212001.

6 M. Meunier, N. Quirke, and A. Aslanides: J. Chem. Phys. 115 (2001) 2876.

7 T. Takada, H. Miyake, Y. Tanaka, and M. Yoshida: Proc. 2014 Int. Symp. Electr. Insul. Mater. (ISEIM) (IEEE, Niigata, 2014) pp. 97-100.

8 T. Takada, H. Kikuchi, H. Miyake, Y. Tanaka, M. Yoshida, and Y. Hayase: IEEE Trans. Dielectr. Electr. Insul. 22 (2015) 1240.

9 A. Huzayyin, S. Boggs, and R. Ramprasad: IEEE Trans. Dielectr. Electr. Insul. 17 (2010) 926.

10 A. Huzayyin, S. Boggs, and R. Ramprasad: IEEE Trans. Dielectr. Electr. Insul. 17 (2010) 920.

11 M. Unge, C. Tornkvist, and T. Christen: IEEE Int. Conf. Solid Dielectr. (ICSD) (IEEE, Bologna, 2013) pp. 935-939.

12 K. Matsui, Y. Tanaka, T. Takada, T. Fukao, K. Fukunaga, T. Maeno, and J. M. Alison: IEEE Trans. Dielectr. Electr. Insul. 12 (2005) 406.

13 Y. Tanaka, R. Kodera, T. Kato, H. Miyake, H. Mori, and Y. Yagi: IEEE Conf. Electr. Insul. Dielectr. Phenom. (CEIDP) (IEEE, Sheraton, 2015) pp. 23-26.

14 W. Wang, D. Min, and S. Li: IEEE Trans. Dielectr. Electr. Insul. 23 (2016) 564.

15 S. Serra, E. Tosatti, S. Iarlori, S. Scandolo, and G. Santoro: Phys. Rev. B 62 (2000) 4389.

16 L. A. Dissado and J. C. Fothergill: Electrical Degradation and Breakdown in Polymers, ed. G. C. Stevens (Peter Peregrinus, London, 1992) Chaps. 1 and 3.

17 M. Righi, S. Scandolo, S. Serra, S. Iarlori, E. Tosatti, and G. Santoro: Phys. Rev. Lett. 87 (2001) 076802. 